\title{
MICROTEXTURE STUDIES OF PST AND PZT CERAMICS AND PZT THIN FILM BY ELECTRON BACKSCATTER DIFFRACTION PATTERNS
}

\author{
C.W. TAI* and K.Z. BABA-KISHI \\ Department of Applied Physics, The Hong Kong Polytechnic University, \\ Hung Hom, Kowloon, Hong Kong
}

(Received 29 June 2001; In final form 20 July 2001)

Crystallographic orientations of twelve different lead scandium tantalate (PST) ceramics, two lead zirconate titanate ceramics (PZT) and one PZT thin film were investigated by the technique of electron backscatter diffraction (EBSD) in the scanning electron microscope. The PST ceramics were processed under different conditions of temperature and annealing. Crystal orientations in the bulk ceramics were examined and the results were plotted in microtexture pole- and inverse pole-figures. Local texture and misorientation between adjacent grains in certain selected regions of the ceramics were also examined. To compare the results of the PST with another lead-based ferroelectric material, an unpoled and a poled PZT ceramics were studied for their texture. The electrical and electromechanical properties of the PZT ceramics were also measured in an attempt to correlate texture with properties. Extensive studies revealed insignificant texture in the ceramics, indicating no relationship between ferroelectricity and texture. Preferred orientations were observed in a PZT thin film grown by pulsed-laser deposition (PLD) method. Though improved anisotropy in PZT thin films can be attributed to texture, there is no indication of the dependence of piezoelectricity on texture.

Keywords: Microtexture; EBSD; Lead scandium tantalate; Lead zirconate titanate; Ferroelectric ceramics; Ferroelectric thin film

\footnotetext{
*Corresponding author. E-mail: apcwtai@polyu.edu.hk
} 


\section{INTRODUCTION}

Most solid-state materials are polycrystalline consisting of grains separated by grain boundaries. Their macroscopic properties are often anisotropic in polycrystalline state (Nye, 1979). Microscopically, each grain, which can be considered as a single crystal, could have its own anisotropic property. A huge number of individual crystallites provide an overall material property which is the integration of the contribution from each grain. Consequently, the materials can have different values of properties or performances if the crystal orientations are different or are changed. Therefore, the orientation is an important factor of the macroscopic properties. Ferroelectricity and pyroelectricity are caused by the spontaneous polarization that occurs in a non-centrosymmetrical crystal structure $(\mathrm{Xu}, 1991)$. The properties of these materials have strong orientation dependence. Besides the dipole in atomic level and domains in microstructure level, the overall texture is also an important factor in the onset of spontaneous polarization.

Crystallographic texture can be defined as the organization of the grains in a polycrystalline material (Bunge, 1994). In microscopic scale, microtexture represents individual crystallite orientation directly (Randle, 1992; Schwarzer, 1993). A convenient experimental method to determine microtexture is the technique of electron backscatter diffraction (EBSD) in scanning electron microscope (SEM). The technique of EBSD, also known as backscatter Kikuchi diffraction pattern (BKDP), involves electron diffraction within a bulk sample in the SEM (Dingley and Randle, 1992) and it is a powerful tool for texture analysis, especially in grain-level study. It utilizes Kikuchi lines and bands for determination of crystal orientations (Randle, 1992; Baba-kishi, 1998). The intensity of the Kikuchi lines can be described by the dynamical theory of electron diffraction (Dudarev et al., 1995). The primary applications of EBSD are micro- and meso-texture determination (Randle, 1992), phase identification by crystallographic point group or space group classification (Baba-kishi and Dingley, 1989; Dingley et al., 1995) and evaluation of plastic and elastic strains (Troost et al., 1993; Wilkinson, 1996). The microtexture results can be typically plotted on pole- and inverse pole-figures to identify preferred orientations and the orientation with respect to the specimen coordinate system, respectively. 
$\mathrm{Pb}_{2}(\mathrm{Sc}, \mathrm{Ta}) \mathrm{O}_{6}$ (PST) is an important member of the order-disorder ferroelectric material, which has been investigated extensively since the compositional heterogeneity model was proposed by Smolensky (1970). When the B-site cations, Sc and Ta, are organized periodically along the [111] directions, the ordered structure is formed. The crystals and ceramics of PST have several system parameters to influence their properties such as dielectric constant, ferroelectricity, pyroelectricity and para-ferroelectric phase transitions. Most of the studies mainly concern the properties related to the degree of structural ordering (Setter and Cross, 1980).

The solid solution of PZT is a well-known ferroelectric material. It has been extensively studied because it has interesting physical properties close to the morphotropic phase boundary (Jaffe et al., 1971). The ceramics of PZT provide an excellent piezoelectric property after applying electrical poling (Xu, 1991). Although PST and PZT have been studied extensively for their properties related to a variety of factors, the influence of crystal orientation on properties has not been studied previously.

In this paper, the crystallographic orientations of twelve different PST ceramics, two PZT ceramics and a PZT thin film, which was prepared by PLD method, were investigated by EBSD in SEM. The average and local textures of PST and PZT ceramics are reported. The texture results are plotted on pole- and inverse pole-figures to determine the preferred orientations with respect to specific directions and specimen coordinate system, respectively.

\section{EXPERIMENTAL}

\section{Materials}

The ceramics of PST were fabricated using mixed-oxide route (Babakishi et al., 1990) and they contained either 5 or $10 \mathrm{wt} . \%$ excess $\mathrm{PbO}$. They were processed under different conditions of temperature and time. Hot-pressing and annealing were also performed. A brief description of their fabrication conditions is shown in Table I.

The PZT ceramics were also prepared by conventional method of mixed-oxide route, corresponding to the composition, $\mathrm{Zr} / \mathrm{Ti}$ is $47 / 53$. 
TABLE I Different fabrication processes for the various samples of PST ceramics used in the present study and the degree of structural order parameter, $\mathrm{S}$

\begin{tabular}{|c|c|c|c|}
\hline Specimen & Excess $\mathrm{PbO}$ & Fabrication Process & $S$ \\
\hline PST(ann-1150) & 5 wt. $\%$ & Annealed at $1150^{\circ} \mathrm{C}$ & 0.86 \\
\hline PST(ann-950) & 5 wt. $\%$ & Annealed at $950^{\circ} \mathrm{C}(6 \mathrm{~h})$ & 0.75 \\
\hline PST(unann- $5 \%$ ) & 5 wt. $\%$ & Unannealed & 0.79 \\
\hline PST(ann1050-5\%) & 5 wt. $\%$ & Annealed at $1050^{\circ} \mathrm{C}(6 \mathrm{~h})$ & 0.80 \\
\hline PST(hp1200-unann) & 10 wt. $\%$ & Hot-press at $1200^{\circ} \mathrm{C}$ and Unannealed & 0.74 \\
\hline PST(hp1200-ann1150) & 10 wt. $\%$ & Hot-press at $1200^{\circ} \mathrm{C}$ and Annealed & 0.72 \\
\hline PST $\left(h p-\mathrm{O}_{2}\right)$ & 5 wt. $\%$ & Controlled $\mathrm{O}_{2}$-atmosphere hot-press & 0.70 \\
\hline PST(unann-10\%) & 10 wt. $\%$ & Unannealed & 0.60 \\
\hline PST(ann1050-10\%) & 10 wt. $\%$ & Annealed at $1050^{\circ} \mathrm{C}(300 \mathrm{~h})$ & 0.65 \\
\hline PST(hp1250-unann) & 5 wt. $\%$ & Hot-press at $1250^{\circ} \mathrm{C}(6 \mathrm{~h})$ and Unannealed & 0.88 \\
\hline PST(hp1050-unann) & 10 wt. $\%$ & Hot-press at $1050^{\circ} \mathrm{C}(6 \mathrm{~h})$ and Unannealed & 0.88 \\
\hline PST(hp950-unann) & 10 wt. $\%$ & Hot-press at $950^{\circ} \mathrm{C}(6 \mathrm{~h})$ and Unannealed & 0.91 \\
\hline
\end{tabular}

One of the PZT ceramics was electrically poled by $4 \mathrm{kV} / \mathrm{mm}$ d.c. at $120^{\circ} \mathrm{C}$ for half an hour.

The PZT thin film was prepared by pulsed-laser deposition (PLD) method, which is described in detail by So et al. (2000). The structure of the multilayer corresponds to $\mathrm{Pb}\left(\mathrm{Zr}_{0.53} \mathrm{Ti}_{0.47}\right) \mathrm{O}_{3}$ (PZT), $\mathrm{La}_{0.7} \mathrm{Sr}_{0.3} \mathrm{MnO}_{3}$ (LSMO) and single crystal $\mathrm{LaAlO}_{3}$ (LAO) as the substrate.

\section{Measurements}

$\mathrm{X}$-ray diffraction was employed to examine the long-range order parameter, $S$, of PST ceramics using standard $\theta-2 \theta$ scan. The intensities of the fundamental (200) and the superstructural (111) reflections were integrated to determine the degree of the structural order (Setter and Cross, 1980). The parameter $S$ for different PST samples is listed in Table I.

To carry out dielectric measurements of the PST ceramics, a thin layer of silver paint was placed on the polished surfaces as electrodes. The dielectric constant was measured at the temperature range $-30-60^{\circ} \mathrm{C}$ and at the frequency of $100 \mathrm{~Hz}$ using an impedance analyzer (HP 4194A) without electrical bias.

For the measurement of the piezoelectric coefficient, $d_{33}$, of the PZT ceramics, the sample surfaces were polished and coated with silver paint as electrodes. The measurements were performed at room temperature using a ZJ-3D PIEZO $d_{33}$-meter (Institute of Acoustics, 
Academia Sinica). The $d_{33}$-meter was calibrated using a standard piezoelectric ceramic of known $d_{33}$ value.

All the microtexture results were obtained using manual EBSD. Prior to the texture study, the ceramics were polished to remove amorphous layers using different grades of diamond pastes. A mixed acid, that is $0.5 \mathrm{Vol} . \% \mathrm{HF}, 4.5 \mathrm{Vol} . \% \mathrm{HCl}$ and $95 \mathrm{Vol} \% \%$ water, was then used to enhance grain boundaries and to remove surface damage. Chemical etching was not performed on the PZT thin film. All the samples were uncoated. Small samples were used to minimize charging due to the primary electron beam and the sample edges were coated with silver paint to disperse electron beam charging. The prepared specimen was mounted onto a specially designed holder that was pre-inclined at an angle $70.5^{\circ}$ to the horizontal. The samples were examined using the Leica 440 scanning electron microscope. The various probe currents used depended on the quality of the diffraction patterns and also on the acquisition time. The EBSD patterns were recorded using a Peltier-cooled low-light CCD of which the screen is parallel to the primary electron beam. The surface topography of the samples was studied using the combined secondary electron and the forward scattered electron detectors. The crystallographic information was plotted on pole- and inverse pole-figures. The misorientations of local texture are presented in the angle/axis notation.

\section{OBSERVATIONS}

\section{PST Ceramics}

The maximum values of dielectric constant range from 2000-11000 as shown in Fig. 1. The various PST samples have very different dielectric responses. Some of the PST samples have broadened paraelectric-toferroelectric transition, indicating their relaxor behavior. The degree of structural ordering of PST samples also varies from 0.65 to 0.91 as listed in Table I.

The average preferred orientations of PST ceramics were examined. Each microtexture pole- and inverse pole-figure represents the results of 600 grains, which were selected randomly in each sample. 


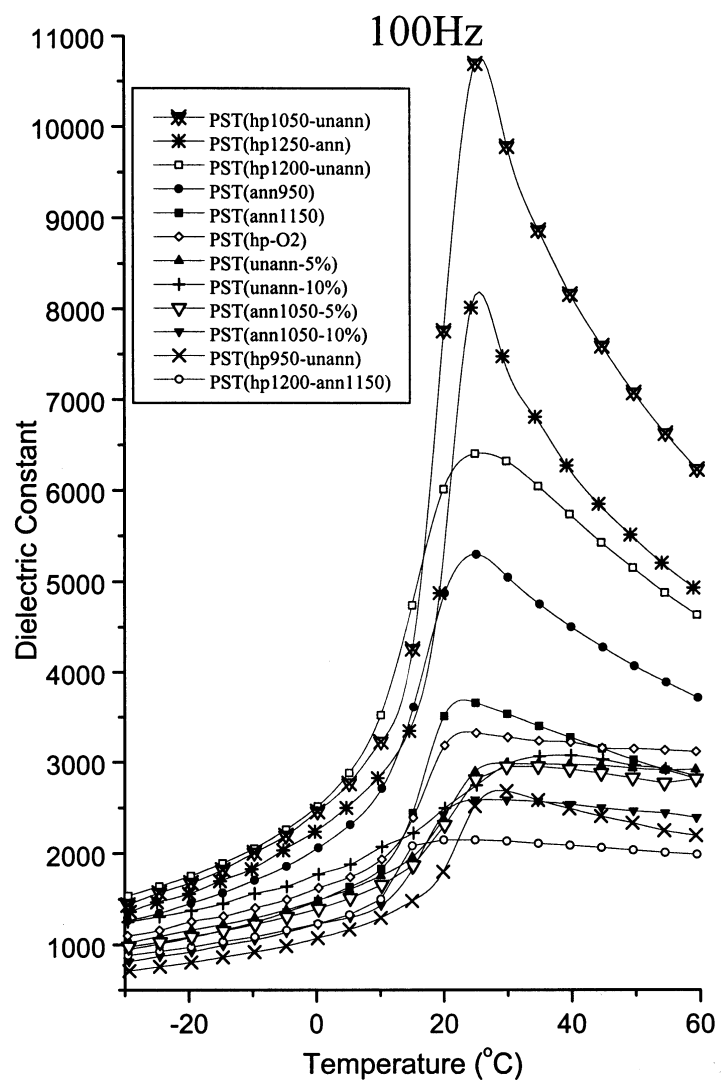

FIGURE 1 The dielectric constants of twelve PST ceramics measured at $100 \mathrm{~Hz}$ under zero bias.

Although the ceramics were fabricated under different conditions (see Table I), their overall orientations were similar and the crystallographic data points were distributed uniformly on the whole figure. The microtexture pole- and corresponding inverse pole-figures of the PST(ann950) sample are shown in Fig. 2. The pole-figures show the $\{002\}$, $\{220\}$ and $\{222\}$ projections, referred to the Fm3m space group. The normal direction (ND), transverse direction (TD) and reference direction (RD) are shown in inverse pole-figures. The data points collected from the other PST samples had similar distribution in both microtexture pole- and inverse pole-figures. Any clustering located 

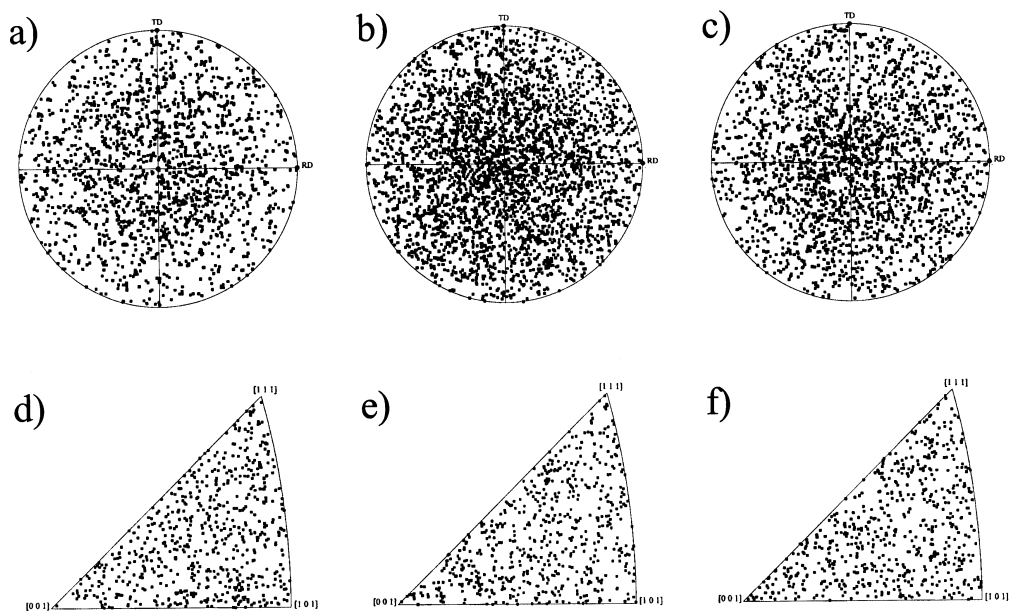

FIGURE 2 (a) $\{002\}$, (b) $\{220\}$ and (c) $\{222\}$ microtexture pole-figures of the PST(ann-950) sample. Each pole-figure contains the results of 600 randomly selected grains. The corresponding ND, RD and TD microtexture inverse pole-figures are shown in (d), (e) and (f), respectively.

on the figure, which indicates the existence of preferred orientation, could not be observed. In conclusion, it was found that these twelve PST ceramics, despite their differing dielectric constants, have random textures.

The PST ceramics often contained large grains. The textures of these large grains were also investigated. The results are plotted in inverse pole-figures, which consist of the orientations of sixty large grains. The ND, RD and TD microtexture inverse pole-figures of the PST(ann-950) sample are shown in Fig. 3. The figure shows no clear indication of the presence of a preferred texture in large grains. The texture of these grains is identical to the overall texture, in which data points are spread out on the figure. Some empty regions were found, indicating the possibility of some preferred orientations in large grains, but the verification could not be performed due to the limited number of these of grains. Consequently, it is concluded that neither preferred orientation nor fiber texture were present in large crystallites.

The PST(ann-950) sample is a representative example of the local texture study in PST ceramics. In this study, 32 grains and 

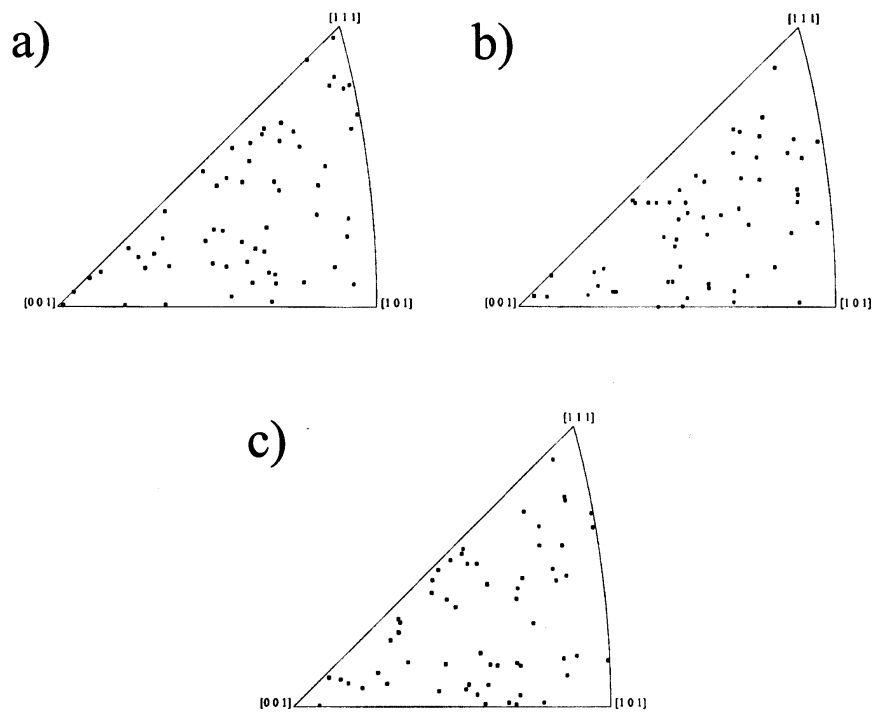

FIGURE 3 (a) ND, (b) RD and (c) TD microtexture inverse pole-figures of the large grains in the PST(ann-950) sample. Each figure contains the results of 60 large grains. No preferred orientation can be observed.

61 misorientations were investigated. In Fig. 4a, a SEM micrograph shows a large grain surrounded by small grains. The large and the surrounding grains were examined for their texture and misorientations. The large grain was $3 \mu \mathrm{m}$ across and was labeled as ' 1 '. The surrounding grains were from 0.25 to $1 \mu \mathrm{m}$ in size. The crystallite orientations are spread out in the inverse pole-figures shown in Figs. 4b, c and d. Fiber texture could not be observed, although there is an empty region in the TD figure, in [111] direction. The relationship between the neighboring grains was also studied. The results are listed in Table II. Most of the misorientations are high-angle grain boundaries and are rotated along random axes. Some low-index axes are found as [011] and [001] in the misorientation of 1-22 and 15-17, respectively. Combining with their misorientation angles, they do not constitute special boundaries. Therefore, the grains are randomly misoriented. There is, therefore, no relationship between the larger grain and the surrounding grains.

In the extensive study of the local texture in PST ceramics, crystallites were randomly oriented and showed no significant relationship 

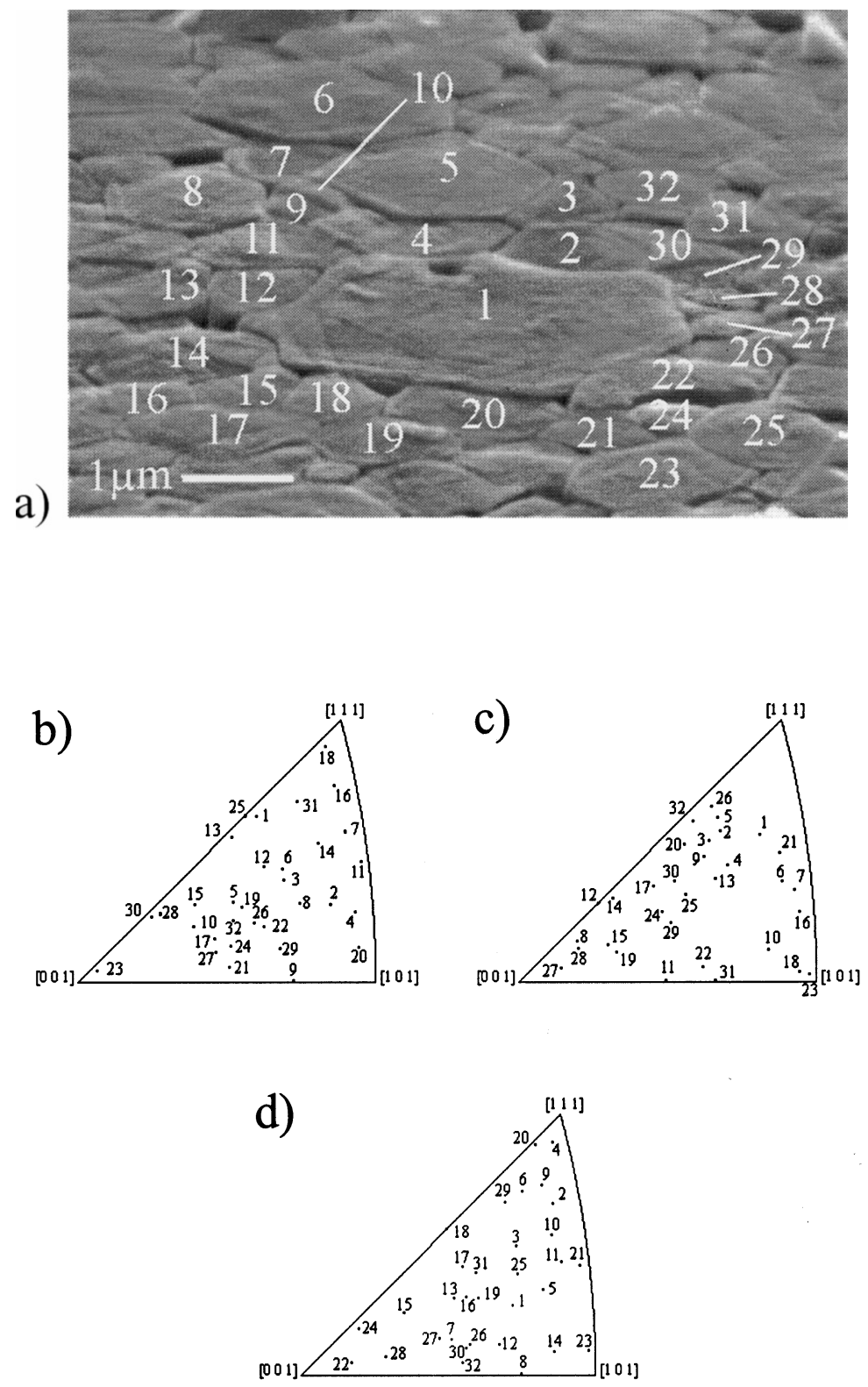

FIGURE 4 (a) SEM image showing a selected region in the PST(ann-950) sample. (b), (c) and (d) are the microtexture inverse pole-figures for the normal, transverse and reference directions of the sample, respectively. 
TABLE II The misorientations of a selected region in PST(ann-950). The misorientations are presented using the angle/axis notation

\begin{tabular}{|c|c|c|c|}
\hline Location & $\theta^{\circ} / U V W$ & Location & $\theta^{\circ} / U V W$ \\
\hline $1-2$ & $48.59 / \overline{4} \underline{5} \overline{9}$ & $8-9$ & $44.37 / \overline{3} 4 \overline{2}$ \\
\hline $1-4$ & $44.52 / 8 \overline{8} 3$ & $8-11$ & $18.30 / 596$ \\
\hline $1-12$ & $41.69 / \overline{2} 23$ & $9-10$ & $50.12 / 1015$ \\
\hline $1-14$ & $36.39 / \overline{1} 23$ & $9-11$ & $46.98 / 691$ \\
\hline $1-15$ & $60.06 / 8 \overline{7} 4$ & $11-12$ & $39.02 / 115 \overline{3}$ \\
\hline $1-18$ & $49.37 / 29 \overline{7}$ & $12-13$ & $48.22 / 1135$ \\
\hline $1-19$ & $49.24 / 9 \overline{2} 7$ & $13-14$ & $42.03 / 934$ \\
\hline $1-20$ & $34.76 / 569$ & $14-15$ & $27.62 / 230$ \\
\hline $1-22$ & $37.78 / 0111$ & $14-16$ & $31.85 / 397$ \\
\hline $1-26$ & $17.78 / \overline{1} 40$ & $15-16$ & $57.05 / 479$ \\
\hline $1-27$ & $55.25 / 4 \underline{3} 4$ & $15-17$ & $15.96 / 001$ \\
\hline $1-28$ & $56.64 / \overline{6} \overline{7} 7$ & $15-18$ & $30.94 / 1116$ \\
\hline $1-29$ & $31.69 / \overline{11} 2$ & $16-17$ & $56.12 / 343$ \\
\hline $1-30$ & $58.35 / \overline{7} 67$ & $18-19$ & $41.96 / \overline{8} 8 \overline{3}$ \\
\hline $2-3$ & $7.83 / 9 \overline{1} \overline{7}$ & $19-20$ & $48.72 / 210$ \\
\hline $2-4$ & $21.16 / \overline{1} 7 \overline{9}$ & $20-21$ & $26.46 / \overline{10} \overline{1} 6$ \\
\hline $2-30$ & $2 8 . 3 1 \longdiv { 1 0 } \overline { 3 } 2$ & $21-22$ & $59.84 / 64 \overline{7}$ \\
\hline $3-4$ & $26.74 / \overline{3} 8 \overline{8}$ & $21-23$ & $27.21 / 010$ \\
\hline $3-5$ & $10.02 / 1 \overline{5} \overline{10}$ & $21-24$ & $45.82 / 934$ \\
\hline $3-32$ & $50.21 / \overline{3} 51$ & $22-24$ & $39.77 / 011 \overline{4}$ \\
\hline $4-5$ & $28.18 / \overline{8} 4 \overline{7}$ & $22-26$ & $37.40 / 6510$ \\
\hline 4-9 & $45.91 / 2 \overline{3} \overline{4}$ & $23-24$ & $42.85 / 962$ \\
\hline $4-10$ & $34.38 / 5 \quad 10 \overline{3}$ & $23-25$ & $45.44 / \overline{5} 96$ \\
\hline $4-11$ & $19.91 / 2 \overline{5} 10$ & $24-25$ & $49.07 / 6011$ \\
\hline $5-6$ & $31.96 / \overline{11} 24$ & $26-27$ & $53.01 / 845$ \\
\hline $5-7$ & $38.11 / 1718 \overline{4}$ & $27-28$ & $11.09 / 0 \overline{3} \overline{2}$ \\
\hline $5-10$ & $43.44 / \underline{1} \overline{0} 0$ & $28-29$ & $49.81 / \overline{2} \underline{\underline{9}}$ \\
\hline $5-11$ & $39.82 / \overline{2} 0 \overline{5}$ & $29-30$ & $54.60 / 0 \overline{8} \overline{7}$ \\
\hline $6-7$ & $35.79 / 58$ & $30-31$ & $53.61 / 5 \overline{8} \overline{6}$ \\
\hline $7-8$ & $33.81 / 11 \overline{2} \overline{3}$ & $31-32$ & $56.44 / \overline{7} \overline{3} 9$ \\
\hline $7-10$ & $54.69 / 289$ & & \\
\hline
\end{tabular}

with their adjacent grains. The high-angle grain boundaries were also highly random.

\section{PZT Ceramics}

In the study of PZT ceramics, the overall and local textures of two samples, one of which was poled and the other unpoled, were examined. The piezoelectric properties and $d_{33}$ coefficients are shown in Table III. The poled PZT had a high piezoelectric coefficient whereas the unpoled PZT showed no piezoelectric property.

The average textures in both the PZT ceramics were investigated. Concerning the average texture, the results were found to be similar 
TABLE III Piezoelectric coefficients, $d_{33}$, of an unpoled and a poled PZT bulk ceramics

\begin{tabular}{ll}
\hline Specimen & $d_{33}(\mathrm{pC} / \mathrm{mm})$ \\
\hline PZT-Unpoled & 0 \\
PZT-Poled & $288 \pm 3$ \\
\hline
\end{tabular}
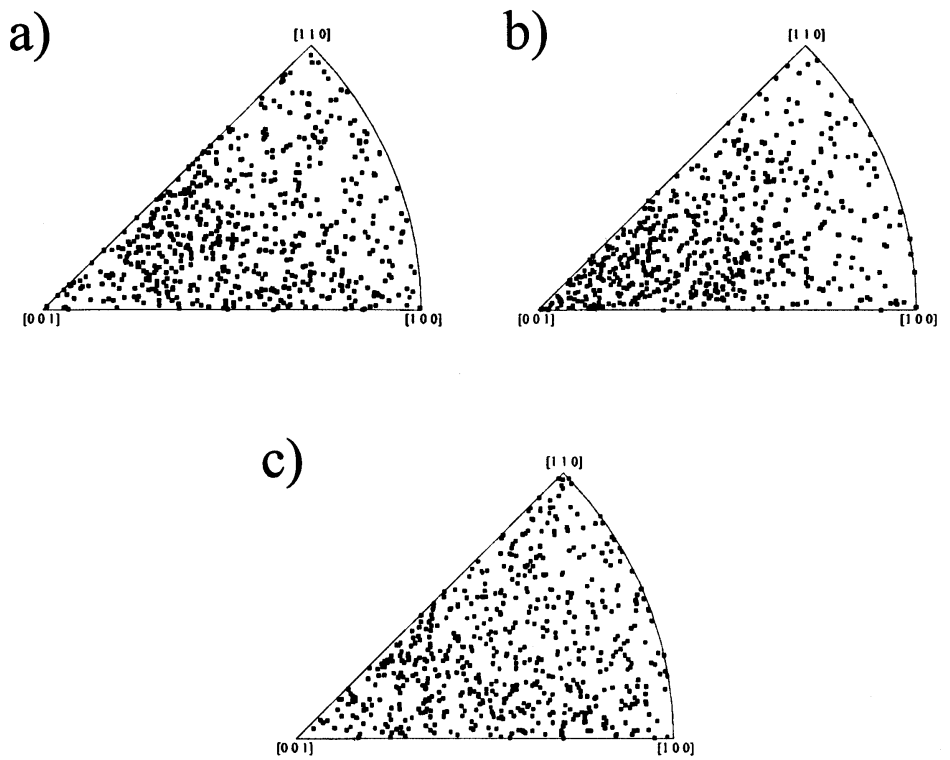

FIGURE 5 (a) ND, (b) TD and (c) RD microtexture inverse pole-figures of the unpoled PZT sample.

to the cases of the PST ceramics in that the data points were distributed randomly on the whole figure. Figures 5 and 6 are the inverse pole-figures of the unpoled PZT and poled PZT, respectively. It can be seen that the samples do not contain any particular texture. However, a slight difference in the texture, shown in the ND inverse pole-figures can be observed qualitatively. The population of the poled PZT near the [001] regions is slightly higher than those in the unpoled PZT; whereas the population of the poled PZT near the [100] region is less than those in the unpoled PZT. We can therefore conclude that the poling of the ceramic has some small influence on crystal orientations. 

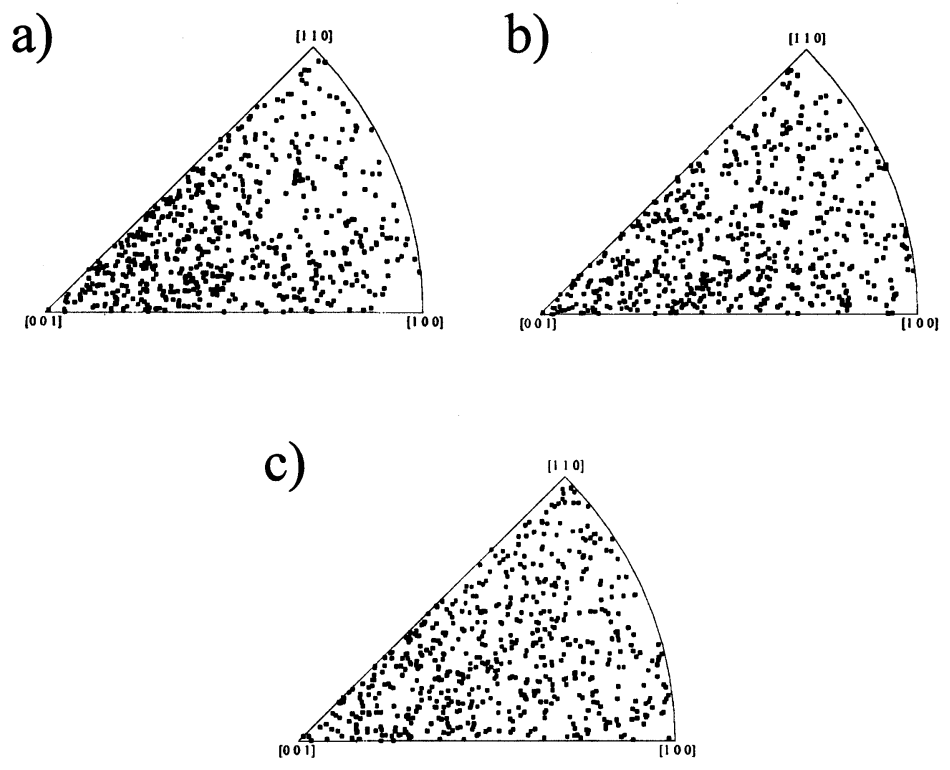

FIGURE 6 (a) ND, (b) TD and (c) RD microtexture inverse pole-figures of the poled PZT sample.

In conclusion, the PZT crystallites were randomly oriented in both the poled and unpoled ceramics. The misorientations studies revealed no special grain boundary configurations. High-angle, random grain boundaries were mostly found in the PZT ceramics.

\section{PZT Thin Film}

The texture of a polycrystalline PZT ceramic thin film grown by PLD method was studied. Crystal orientations were plotted on the microtexture pole- and inverse pole-figures. Figure 7 is the microtexture pole- and corresponding inverse pole-figures of the PZT ceramic thin film recorded from about 100 grains. The microtexture pole-figures, $\{100\},\{110\}$ and $\{111\}$, show highly preferred orientations, that resemble fiber-like texture. The $\{100\}$ pole-figure shows an orientation cluster, which is located at the center of the figure. The other three clusters are located at the circumference. The clustering at the center originates from two groups of orientations, 

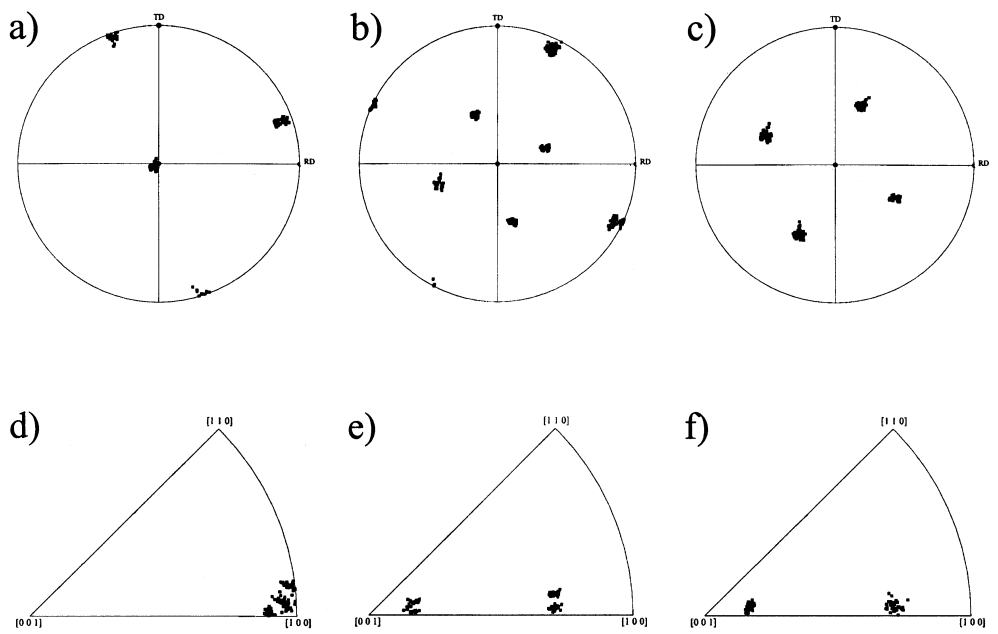

FIGURE 7 Microtexture pole-figures of the PZT ceramic thin film. The three directions, (a) $\{100\}$, (b) $\{110\}$ and (c) $\{111\}$ projections are plotted. The corresponding microtexture ND, TD and RD inverse pole-figures are shown in (d), (e) and (f), respectively. The ND figure shows the normal direction of the film pointing to [100]. The RD and TD figures show the other two directions of the film oriented along [013] and [031].

which are rotated by $90^{\circ}$ along the [001] direction. The clusters are not formed at the intersection of the circumference and the straight lines because the sample was arbitrarily placed with respect to the transverse and reference directions. In the $\{110\}$ projection shown in Fig. $7 \mathrm{~b}$, the data can be divided into four texture groups that are $\{100\}\langle 0 \overline{3} \overline{1}\rangle,\{100\}\langle 0 \overline{3} 1\rangle,\{100\}\langle 013\rangle$ and $\{100\}\langle 031\rangle$. The presence of four groups is caused by the cubic symmetry. In Fig. 7d, the ND figure shows the normal component of the grain orientations. Three groups can be observed close to the [100] direction. Their angular difference is smaller than about $5^{\circ}$. Two main groups, which can be seen in Figs. 7e and $\mathrm{f}$ are located at [013] and [031]. These inverse pole-figures confirmed the existence of highly textured PZT crystallites. The crystallographic texture of the PZT ceramic thin film can be expressed by $\{100\}\langle 031\rangle$ from the pole- and inverse pole-figures. Since the reference and transverse directions are placed arbitrarily, the $\mathrm{RD}$ or TD can be rotated at an angle along the normal direction [100]. As a result, the general expression of the thin film texture can be re-written as $\{100\}\langle 001\rangle$. 


\section{DISCUSSION}

All the twelve PST ceramics show no preferred orientations either in average or local texture. The crystallographic texture results, that are similar to the studies on the coefficient of electrostriction $\mathrm{Q}_{11}$ (Birks et al., 1999) and the general profile of temperature dependence refractive index $\delta n(T)$ (Korshunov et al., 1989), are not significantly related to the degree of structural order. In addition, the variations in dielectric constants of PST materials are not influenced by the existence of random texture. The local texture results in PST ceramics showed high-angle random boundaries. It is an essential condition to promote higher diffusivity along and near the grain boundaries. It may be the most probable explanation for the presence of enhanced structurally ordered domains near grain boundaries in large grains and the higher degree of structural order in small grains (Randall et al, 1986; Baba-kishi and Barber, 1990). In the local texture study, the large and small grains have no preferred orientations. The misorientations show that the large grain do not influence the surrounding grains.

In the PZT ceramics, highly preferred orientations are also absent. However, slight differences in the crystallographic orientations were detected qualitatively, by observing the distribution in the microtexture inverse pole-figures. The differences are related to the effect of poling.

The preferred texture in the PZT film along the [100] directions was clearly evident in both the microtexture pole- and inverse pole-figures. The PZT crystallites were highly oriented along $\{100\}\langle 001\rangle$, which is produced by a suitable substrate with lattice matching. The substrate provides a template for the crystal growth, which needs to be controlled to maintain the texture and the size of crystallites. However, the use of PLD is not essential for obtaining texture.

\section{CONCLUSION}

Microtexture investigations of twelve ceramics specimens of the ferroelectric PST ceramics, two PZT ceramics and a PZT polycrystalline ceramic thin film were carried out by the technique of EBSD in 
SEM. The PST and PZT ceramics showed no preferred orientation in average and local texture studies. Consequently, the specific anisotropic properties of the ceramics that are dielectric constant in PST and piezoelectric coefficient in PZT, are not under the influence of texture. Small change in orientations was observed in PZT ceramics after electrical poling. The local texture results of the PST ceramics provide the first experimental evidence of the grain boundary conditions that explain the configurations of the structurally ordered domains. A PZT multilayer thin film was shown to be highly textured. In all the samples studied, we have seen no evidence of any relationship between the electrical properties and the crystallographic texture.

\section{Acknowledgements}

This work is supported by the Hong Kong Polytechnic University through the research grant G-V714. One of the authors (C.W. Tai) is grateful for the award of a research studentship from the Hong Kong Polytechnic University. The sample of the PZT thin film was provided by Dr. K.H. Wong.

\section{References}

Baba-kishi, K.Z. and Dingley, D.J. (1989). Backscatter Kikuchi diffraction in the SEM for identification of crystallographic point groups. Scanning, 11, 305-312.

Baba-kishi, K.Z. and Baber, D.J. (1990). Transmission electron microscope studies of phase transitions in single crystals and ceramics of ferroelectric $\mathrm{PbSc}_{1 / 2} \mathrm{Ta}_{1 / 2} \mathrm{O}_{3}$. Journal of Applied Crystallography, 23, 43-54.

Baba-kishi, K.Z., Reaney, I.M. and Barber, D.J. (1990). Transmission electron microscopy of second-phase particles in $\mathrm{Pb}\left(\mathrm{Sc}_{1 / 2} \mathrm{Ta}_{1 / 2}\right) \mathrm{O}_{3}$ ferroelectric ceramics. Journal of Materials Science, 25, 1645-1655.

Baba-kishi, K.Z. (1998). Measurement of crystal parameters on backscatter Kikuchi diffraction patters. Scanning, 20, 117-127.

Birks, E., Sternberg, A. and Shebanovs, L. (1999). Effect of structure ordering in complex ferroelectric perovskite. Ferroelectrics, 223, 107-111.

Bunge, H.J. (1994). Statistical crystallography of the polycrystal. Materials Science Forum, 157-162, 13-30.

Dingley, D.J. and Randle, V. (1992). Microtexture determination by electron backscatter diffraction. Journal of Materials Science, 27, 4545-4566.

Dingley, D.J., Baba-kishi, K.Z. and Randle, V. (1995). Atlas of Backscattering Kikuchi Diffraction Patterns. The Institute of Physics, Bristol.

Dudarev, S.L., Rez, P. and Whelan, M.J. (1995). Theory of electron backscattering from crystals. Physical Review B, 51, 3397-3412.

Jaffe, B., Cook, W.R. and Jaffe, H. (1971). Piezoelectric Ceramics. Academic Press, London. 
Korshunov, O.Y., Markovin, P.A., Pisarev, R.V. and Sapozhnikov, L.M. (1989). Thermooptical properties of ferroelectric lead scandium tantalate $\mathrm{PbSc}_{0.5} \mathrm{Ta}_{0.5} \mathrm{O}_{3}$ with a different degree of ordering. Ferroelectrics, 90, 151-153.

Nye, J.F. (1979). Physical Properties of Crystals. Oxford University Press, Claredon.

Randall, C.A., Barber, D.J., Whatmore, R.W. and Groves, P. (1986). A TEM study of ordering in the perovskite $\mathrm{PbSc}_{1 / 2} \mathrm{Ta}_{1 / 2} \mathrm{O}_{3}$. Journal of Materials Science, 21, 4456-4462.

Randle, V. (1992). Microtexture Determination and Its Application. The Institute of Materials, London.

Schwarzer, R.A. (1993). The determination of local texture by electron diffraction. Textures and Microstructures, 20, 7-27.

Setter, N. and Cross, L.E. (1980). The role of B-site cation disorder in diffuse phase transition behavior of perovskite ferroelectrics. Journal of Applied Physics, 51, 4356-4360.

Smolensky, G.A. (1970). Physical phenomena in ferroelectrics with diffused phase transition. Journal of the Physical Society of Japan, 28(Suppl.), 26-37.

So, K.S., Wong, K.H. and Wu, W.B. (2000). Thermal stability and semiconducting properties of epitaxial $\mathrm{La}_{0.7} \mathrm{Sr}_{0.3} \mathrm{MnO}_{3}$ films. Materials Research Society Symposium Proceedings, 623, 77-82.

Troost, K.Z., Sluis P. and Gravesteijn, D.J. (1993). Microscale elastic-strain determination by backscatter Kikuchi diffraction in the scanning electron microscope. Applied Physics Letters, 62, 1110-1112.

Wilkinson, A.J. (1996). Measurement of elastic strains and small lattice rotations using electron back scatter diffraction. Ultramicroscopy, 62, 237-247.

Xu, Y. (1991). Ferroelectric Materials and Their Applications. North-Holland, Amsterdam. 